\title{
Delays in Care Associated With Non-English-Speaking Patients With Breast Cancer
}

Katy E. Balazy, MD, MPH${ }^{1}$; Cecil M. Benitez, MD, $\mathrm{PhD}^{2}$; Paulina M. Gutkin, BS ${ }^{3}$; Clare E. Jacobson, BA ${ }^{4}$; Rie von Eyben, $\mathrm{MS}^{1}$; and Kathleen C. Horst, $\mathrm{MD}^{1}$

\begin{abstract}
Background: Breast cancer care requires coordination between multiple diagnostic and treatment modalities. Disparities such as age, race/ ethnicity, and socioeconomic status are associated with delays in care. This study investigates whether primary language is associated with delays in breast cancer diagnosis and treatment before and through radiotherapy (RT). Patients and Methods: This study was an institutional retrospective matched-cohort analysis of women treated with breast RT over 2 years. A total of 65 non-English-speaking (NES) patients were matched with 195 English-speaking (ES) patients according to stage, age, and chemotherapy delivery. Key time intervals along the breast cancer care path from initial findings through RT were recorded. Data were analyzed in a mixed model with matching as the random effect. The impact of race and insurance status was analyzed in addition to language. Results: Significant delays were found for NES patients, which varied by race. NES Latina patients experienced the longest delay, with a mean total care-path time of 13.53 months (from initial findings to end of RT) versus 8.18 months for all ES patients $(P<.0001)$. Specifically, their mean total care-path time was 5.97 months longer than that of ES Latina patients $(P=.001)$ and 5.80 months longer than that of ES White patients $(P<.0001)$. In addition, NES Latina patients had a significantly longer total care-path time than NES patients of other races/ethnicities $(P=.001)$. Delays were specifically seen between initial clinical or radiographic findings and diagnostic mammogram $(P=.001)$ and between biopsy and resection $(P=.044)$. Beyond language, race/ethnicity was itself associated with delays between resection and start of RT $(P=.032)$ and between start and end of RT $(P=.022)$. Conclusions: Language is associated with pre-RT delays in breast cancer care, especially for NES Latina patients. Delays are most pronounced before diagnostic mammograms, but they also exist before resection and RT. Future work should target NES patients to assist their progress along the care path.
\end{abstract}

J Natl Compr Canc Netw, doi: 10.6004/jnccn.2020.7797 Published online August 10, 2021

${ }^{1}$ Department of Radiation Oncology, Stanford Comprehensive Cancer Center, Stanford University School of Medicine, Stanford, California; ${ }^{2}$ Highland Hospital, Oakland, California; ${ }^{3}$ Medical College of Wisconsin, Milwaukee, Wisconsin; and

${ }^{4}$ University of Michigan Medical School, Ann Arbor, Michigan.

\section{Background}

Breast cancer is the second leading cause of cancer death for women in the United States, with an estimated 276,480 new diagnoses and 42,170 breast cancer-related deaths in $2020 .^{1}$ Despite mortality declining by $40 \%$ between 1989 and 2017, disparities in care, including those related to race/ethnicity, sex, age, insurance, marital status, income, education, and geographic location, continue to be a major concern. ${ }^{1-5}$

Disparities impact both presentation and outcomes for patients with breast cancer. ${ }^{6-8}$ Cancer incidence among Black and Hispanic patients continues to increase, ${ }^{8}$ and survival rates are $9 \%$ lower for Black women than for White women in the United States. ${ }^{1}$ Low socioeconomic status is associated with later-stage disease, larger tumors, and decreased survival even for those enrolled in Medicare/ Medicaid. ${ }^{7}$ Tumor biology and genetics also contribute to disparities. Some portion of increased mortality seen in Black women is attributed to an increased risk of aggressive triple-negative breast cancer, but this statistic has not been shown for Hispanic women. ${ }^{9}$ However, biologic and genetic differences do not fully account for the more advanced-stage presentation in Black and Hispanic women. ${ }^{10}$ Sociodemographic deprivation is thought to influence disparities in addition to tumor biology. ${ }^{11}$

Breast cancer care requires a high level of coordination, including mammograms, biopsies, consults, surgical resection, and often chemotherapy and radiation. Delays in care can risk patient outcomes at any of these timepoints. Race/Ethnicity disparities have been shown to impact delays in diagnosis and treatment. ${ }^{12,13}$ In women aged $<50$ years, Black women experienced greater delays than White women. ${ }^{14}$ Similar findings have been seen in patients of older age, those who are unmarried, or those from a lower socioeconomic background. ${ }^{15,16}$

Language barriers are an important disparity that have only been studied in a limited capacity. Non-Englishspeaking (NES) patients have been shown to present

See JNCCN.org for supplemental online content. 
with later-stage breast cancer than their English-speaking (ES) counterparts. ${ }^{17}$ Given that non-English use in the United States increased nearly $150 \%$ from 1980 to $2009,{ }^{18}$ effective communication is increasingly important to provide high-quality healthcare. However, $<50 \%$ of surveyed oncologists and surgeons reported the use of professional interpretation services, and more than half reported difficulty in discussing treatment options and prognosis with NES patients. ${ }^{19,20}$

Although communication is of paramount importance in providing patient-centered care, the impact of language on delays in breast cancer care is largely unknown. In this study, we sought to determine whether primary language impacts delays in care for a cohort of women undergoing breast radiotherapy (RT) - from initial clinical or radiographic findings through diagnostic mammogram, biopsy, and treatment. We hypothesized that treatment delays would disproportionally impact NES patients.

\section{Patients and Methods}

\section{Patients and Location}

An institutional retrospective matched-cohort analysis was performed on all women diagnosed with breast cancer and treated with RT from 2016 through $2017(\mathrm{~N}=418)$. Demographic data including age, primary language, insurance, and race/ethnicity were obtained from electronic medical records. NES patients were defined as those who required an interpreter and/or who designated their primary language as one other than English. The most represented languages were Spanish, Mandarin, and Vietnamese (supplemental eTable 1, available with this article at JNCCN.org). Patients were classified into 4 race/ethnicity categories: White, Asian, Latina, and Black/other. Stage of breast cancer (0/ductal carcinoma in situ [DCIS], I, II, III, and IV) was recorded based on physician documentation at patient presentation. Health insurance was categorized as public if it was through Medicaid or Medicare and as private for all other insurance, including self-pay. There was no patient navigation program for this cohort of patients. Interpreters were available by video or in person when requested.

Data were gathered from a single academic institution in California. The California population has a high representation of NES patients-18.8\% of California's households have limited English proficiency versus $8.2 \%$ of households nationwide. ${ }^{21-23}$ The most represented languages in California are Spanish (64.8\%), Chinese (Cantonese or Mandarin, 7.7\%), and Vietnamese (3.4\%). Similar to California at large, this study cohort included $20.11 \%$ NES patients. This study population had fewer Spanish speakers and more Mandarin/Vietnamese speakers than statewide numbers $(42.9 \%$ Spanish, $14.3 \%$ Mandarin, and $11.4 \%$ Vietnamese). Note that there are differences in healthcare and insurance coverage between states. California is 1 of 39 states to have accepted Medicaid expansion under the Affordable Care Act, and covered California serves as a statewide marketplace. ${ }^{24,25}$

\section{Matching}

Matching between ES and NES patients was performed using propensity score matching, with each NES patient matched with at most $5 \mathrm{ES}$ patients. Each match was based on the criteria of stage, age, and chemotherapy. These factors were chosen for matching because they are wellknown to impact treatment time: later-stage disease often requires chemotherapy and a more extensive treatment course, older adult age can lead to more limited treatment recommendations, and chemotherapy adds significant time in either the neoadjuvant or adjuvant setting. Patients in the study had to match on all 3 factors: an exact match of stage (0/DCIS, I, II, III, or IV), an exact match on chemotherapy (neoadjuvant, adjuvant, or neither), and a match on age within a range of \pm 5 years. Given the sample size (260 women treated for breast cancer) and the desire to test race/ethnicity and insurance as individual predictors, matching was not performed on race/ethnicity and insurance. Instead, these 2 predictors were included alongside language as independent predictors in the mixed model as described later.

\section{Statistics}

Patients were matched based on the criteria described earlier. The key time intervals in the breast cancer care path were designated as follows: the interval from initial findings (palpable mass or abnormal screening mammogram) until diagnostic mammogram, from diagnostic mammogram until biopsy, from biopsy until surgical resection, from surgical resection until start of RT, and from start until end of RT, and the total interval from initial findings until end of RT (total care-path time). Patients were matched on chemotherapy; it was not included as a separate time interval. Only patients with the same chemotherapy status (neoadjuvant, adjuvant, or neither) were compared, and all increases in time intervals are after accounting for chemotherapy.

Next, each interval was analyzed in a separate mixedeffects model. A mixed-effects model (also referred to as a mixed model or mixed error-component model) is a type of regression model that allows for clustering of patients based on known effects (in this study, age/stage/chemotherapy), and then analyzes patients within and between clusters based on individual predictors (language, race/ ethnicity, and insurance). Because it is known that stage, age, and chemotherapy impact treatment time, patients were matched and clustered based on these factors. However, it was not established whether language, race/ethnicity, and insurance impacted treatment time within and 
between these clusters. At each time interval, these clusters were analyzed in a mixed-effects regression analysis to determine whether the individual predictors of language (English vs non-English), race/ethnicity, and insurance were significantly associated with time. All potential interactions were examined, and only the interaction of language and race/ethnicity was included because all the others were not significant. Including these individual predictors allowed us to test whether each predictor impacted time while simultaneously accounting for the other predictors. When a predictor was significant, the implication was that the predictor had an impact on the outcome either instead of or in addition to the other predictors in the model. All statistical analyses were performed using SAS version 9.4 (SAS Institute Inc).

\section{Results}

Over 2 years (2016 and 2017), a total of 418 women with breast cancer were treated with RT at a single institution, including 70 NES and 348 ES patients. Of these, 65 NES patients were matched successfully on all 3 matching criteria (age, stage, and chemotherapy) to 195 ES patients, for a total cohort of 260 (supplemental eFigure 1).

Average age of the cohort was 57.30 years (SD, 10.58). Most patients had early-stage disease (0/DCIS or I, $65.38 \%)$. There were borderline significant differences in stage $(P=.058)$ and chemotherapy $(P=.053)$ between ES and NES patients, which were controlled for by propensity score matching between the groups (Table 1). Most patients in this study were White $(42.3 \%)$, followed by Asian (28.8\%), Black/other (15.8\%), and Latina (13.1\%). There was an expected and significant difference between ES and NES patients in terms of insurance status and race/ethnicity. ES patients were more likely to have private insurance $(71.3 \%$ vs $27.7 \%$ of NES patients; $P<.0001)$. Although most ES patients were White $(52.8 \%$; $P<.001)$, most NES patients were Asian $(40.00 \%)$ and Latina (36.9\%; $P<.0001$ ) (Table 1). This cohort consisted of patients who underwent RT, and therefore most received breast-conservation surgery $(96.9 \%)$ versus mastectomy; there was no significant difference in surgery type between NES and ES patients $(P=.113)$. The range of prescribed radiation courses was 10 to 33 treatments, and there was no difference between NES and ES patients $(P=.794)$.

Of the 5 time intervals analyzed, the longest interval was from resection to start of RT (mean [SD], 2.8 [1.8] months), and the shortest was from diagnostic mammogram to biopsy (mean [SD], 0.5 [0.9] months). The mean (SD) total care-path time was 8.3 (5.2) months (Figure 1A).

\section{Association Between Language and Delays in Care} For total care-path time (initial clinical or radiographic findings to end of RT), the interaction between primary

\begin{tabular}{|c|c|c|c|}
\hline Characteristic & $\begin{array}{l}\text { ES } \\
n(\%)^{a}\end{array}$ & $\begin{array}{l}\text { NES } \\
\text { n (\%) }\end{array}$ & $P$ Value $^{\mathrm{b}}$ \\
\hline Total, $\mathrm{n}$ & 195 & 65 & \\
\hline $\begin{array}{l}\text { Mean age } \\
(95 \% \mathrm{Cl}), \mathrm{y}\end{array}$ & $\begin{array}{l}57.07 \\
(55.6-58.5)\end{array}$ & $\begin{array}{l}58.00 \\
(55.0-61.0)\end{array}$ & $.54^{c}$ \\
\hline Stage & & & $.058^{c}$ \\
\hline 0 & 47 (24.1) & 14 (21.5) & \\
\hline I & $88(45.1)$ & $21(32.3)$ & \\
\hline II & $46(23.6)$ & $19(29.2)$ & \\
\hline III & $14(7.2)$ & $11(16.9)$ & \\
\hline Chemotherapy & & & $.053^{\mathrm{c}}$ \\
\hline None & $150(76.9)$ & $40(61.5)$ & \\
\hline Neoadjuvant & $22(11.3)$ & $12(18.5)$ & \\
\hline Adjuvant & $23(11.8)$ & $13(20.0)$ & \\
\hline Insurance status & & & $<.0001^{d}$ \\
\hline Private & $139(71.3)$ & $18(27.7)$ & \\
\hline Public & $56(28.7)$ & 47 (72.3) & \\
\hline Race/Ethnicity & & & $<.0001^{d}$ \\
\hline White & $103(52.8)$ & $7(10.8)$ & \\
\hline Asian & $49(25.1)$ & $26(40.0)$ & \\
\hline Latina & $10(5.1)$ & $24(36.9)$ & \\
\hline Black/Other & $33(16.9)$ & $8(12.3)$ & \\
\hline Surgical resection & & & .113 \\
\hline Mastectomy & $4(2.1)$ & $4(6.2)$ & \\
\hline Lumpectomy & $191(97.9)$ & $61(93.8)$ & \\
\hline \multicolumn{4}{|l|}{ Radiation treatment } \\
\hline $\begin{array}{l}\text { Mean fractions } \\
(95 \% \mathrm{Cl})\end{array}$ & $\begin{array}{l}20.2 \\
(18.2-21.3)\end{array}$ & $\begin{array}{l}20.0 \\
(18.3-21.6)\end{array}$ & .794 \\
\hline Received a boost & $99(51.3)$ & $39(60.0)$ & .224 \\
\hline
\end{tabular}

Bold indicates statistically significant $P$ value.

Abbreviations: ES, English-speaking; NES, non-English-speaking.

apercentages have been rounded and may not total 100 .

${ }^{b}$ Age was calculated using the $t$ test; for other comparisons, the chi-square was used.

'Age, stage, and chemotherapy were the 3 factors in the matching algorithm. Patients needed to match on all 3 to be included. Therefore, these noted differences between the NES and ES cohorts in regard to stage and chemotherapy were controlled for via propensity score matching.

${ }^{d}$ Race/Ethnicity and insurance status differences were controlled for by including them as individual predictors in the mixed-effects regression model.

language and race/ethnicity was significant in the mixed-effects model $(P=.024)$. This finding indicates that language impacted the total care path differently depending on a patient's race/ethnicity (supplemental eTables 2-6). The NES Latina patients had the longest mean total care path of 13.53 months, compared with 8.18 months for all ES patients $(P<.0001)$. They had a 5.97-month delay compared with ES Latina patients $(P=.001)$ and a 5.80-month delay compared with ES White patients $(P<.0001)$ (Figure $1 C$, Table 2$)$. In addition, NES Latina patients had a significantly longer total care-path time than NES patients of other races/ethnicities (13.53 months vs 9.59 months for Black/other 
A

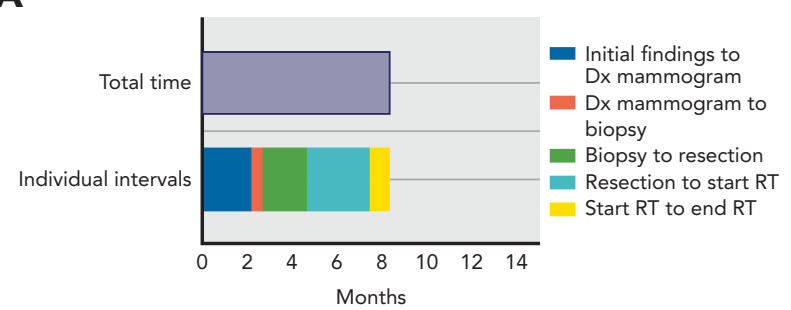

B

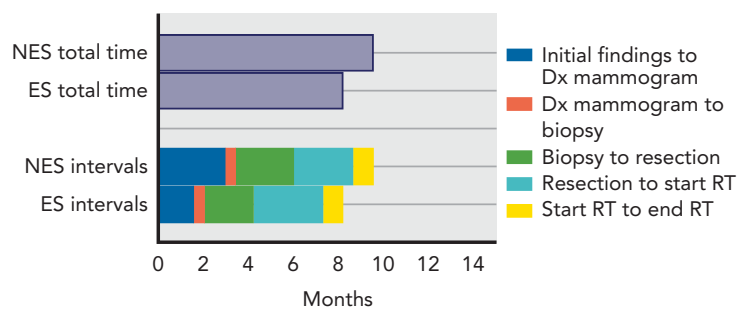

C

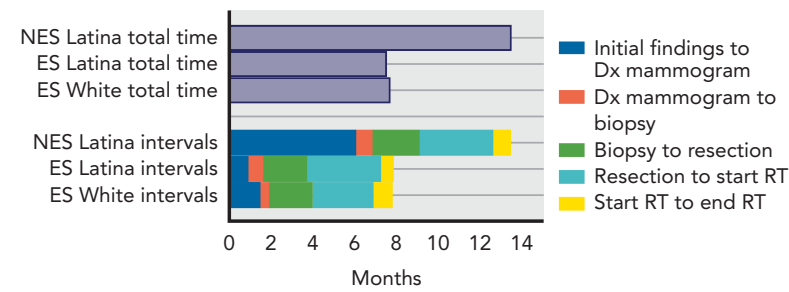

Figure 1. Mean time (months) between cancer care intervals for (A) all matched patients, (B) NES versus ES patients (interval lengths are listed in Table 3), and (C) NES Latina patients versus ES Latina and ES White patients (interval lengths are listed in Table 2).

Statistical methods: (A) Time was determined with a simple mean calculation using all patients in the matched cohort; standard deviations were then separately calculated for this cohort. (B, C) Time intervals are predicted means from the mixed-effects model, with accompanying $P$ values.

Abbreviations: Dx, diagnostic; ES, English-speaking; NES, non-Englishspeaking; RT, radiotherapy.

patients, 8.25 months for Asian patients, and 7.48 months for White patients) (Figure 2A, supplemental eTable 5).

Pre-RT delays for NES patients were observed at 2 specific intervals: between initial clinical or radiographic findings and diagnostic mammogram, and between biopsy and resection (Figure 1B). There was a difference between NES and ES patients in regard to presenting with palpable versus screening findings; significantly more NES patients presented with palpable findings $(47.7 \%)$ versus ES patients $(28.4 \% ; P=.0042)$. For this interval between initial findings and diagnostic mammogram, the interaction between primary language and race/ethnicity again was significant $(P=.014)$. NES Latina patients showed the longest mean time of 6.09 months, versus 1.60 months for all ES patients $(P<.0001)$. Compared with ES Latina patients, NES Latina patients had a 5.16-month delay $(P<.0006)$ and a 4.59-month delay compared with ES White patients $(P<.0001)$ (Figure 1C, Table 2). There was also a longer delay for NES Latina patients than NES patients of other races/ethnicities $(P=.001): 6.09$ months for NES Latina patients versus 3.02 months for NES Black/other patients, 2.27 months for NES Asian patients, and 0.61 months for NES White patients (Figure 2B). Notably, NES Black/other patients also had a longer interval of 3.02 months, a 1.41-month delay compared with that of ES Black/other patients $(P=.360)$, and a 1.52-month delay compared with that of ES White patients $(P=.291)$. Although neither of these comparisons was statistically significant, the results may be because of the limited sample size in the Black/ others cohort.

NES patients experienced another pre-RT delay, with a longer time interval between biopsy and resection compared with ES patients $(P=.044)$ (Table 3$)$. Race/Ethnicity was not significant at this interval $(P=.690)$, nor was the interaction between language and race/ethnicity $(P=.803)$ (supplemental eTable 2). NES patients took 2.61 months from biopsy to resection compared with ES patients, who took 2.20 months (Figure 1B).

Notably, insurance status was not significant at any time interval, nor was any interaction between insurance and other predictors (supplemental eTables 2 and 6).

Association Between Race/Ethnicity and Delays in Care There were 2 time intervals for which race/ethnicity was a significant indicator of delay but primary language was not: resection to start of RT, and start to end of RT. Latina patients averaged 3.58 months between resection and start of RT, versus 2.60 to 2.76 months for Asian, White, and Black/other patients $(P=.035)$. Latina patients then experienced an inverse relationship during their RT course and had a shorter treatment time than patients of other races/ethnicities $(P=.0313)$ : 23.44 days for Latina patients versus 27.7 days for White patients. Meanwhile, Black/ other patients (30.44 days) and Asian patients (29.53 days) showed a slightly longer RT treatment time than White patients (27.7 days).

\section{Discussion}

Delays in diagnosis and treatment have been a focus of quality breast cancer initiatives. ${ }^{26}$ Delays portend worse breast cancer outcomes, including later stage at diagnosis and decreased overall survival (OS) ${ }^{27}$ A delay between diagnosis and first treatment of $>90$ versus $<30$ days has indicated worse OS, with a hazard ratio of $2.25 .{ }^{28}$ One large-scale study of $>420,000$ patients with breast cancer revealed that $>12$ weeks between diagnosis and surgery was associated with decreased OS. ${ }^{29}$ A SEER Medicare analysis of $>94,000$ patients showed that delays between diagnosis and surgery were associated with lower OS and that breast cancer-specific mortality increased with each 60-day interval. ${ }^{30}$ 
A

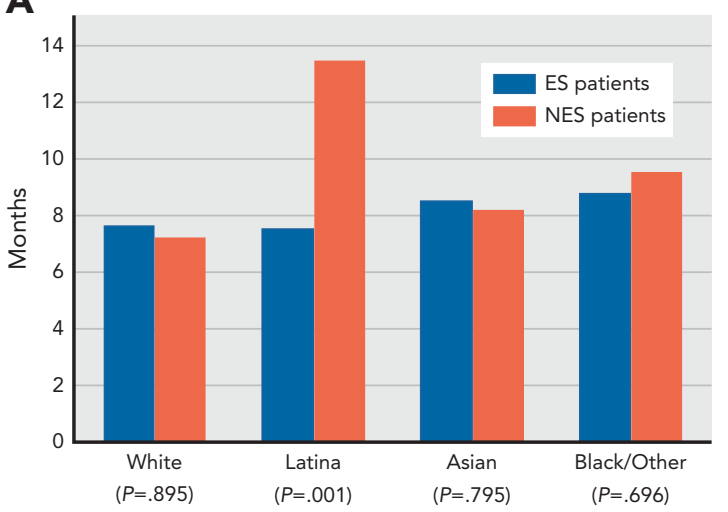

B

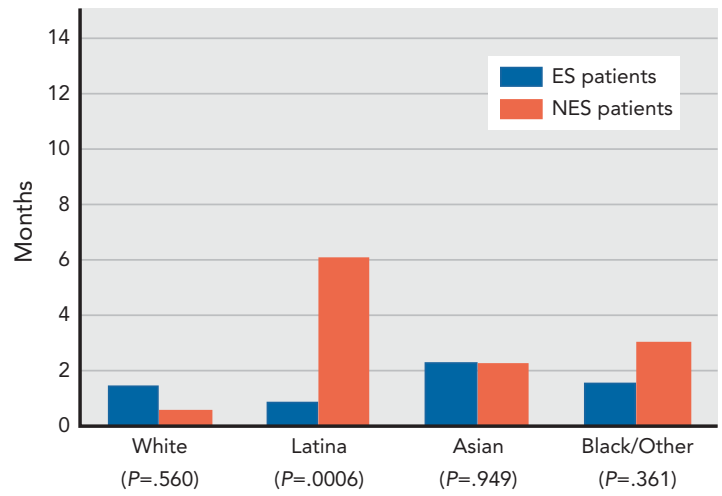

Figure 2. Time intervals by race/ethnicity for (A) total care path and (B) initial findings to diagnostic mammogram. Statistical methods: Time intervals are predicted means from the mixed-effects model, with accompanying $P$ values. Abbreviations: ES, English-speaking; NES, non-English-speaking.

Patient disparities contribute to delays and include demographic factors such as older age, living in rural areas, and lower levels of education. ${ }^{31}$ Race/Ethnicity has a significant correlation with delays in care. ${ }^{32}$ Black patients have experienced longer delays than patients of other races. ${ }^{13} \mathrm{~A}$ SEER analysis of nearly 50,000 women in the United States showed that Black women experienced the greatest delays in both diagnosis and initiation of treatment, with a 1.64 odds of delay beyond 1 month. ${ }^{33}$

Little has been studied in regard to primary language and delays in care. One study by Khanna et $\mathrm{al}^{13}$ included primary language alongside race/ethnicity, insurance status, cancer stage, and marital status in an institutional retrospective multivariate analysis on breast cancer treatment delay. They did not find a significant association between language and delays in care, although it is not documented whether potential interactions between race and primary language were examined. The study's main measure was time from biopsy to first treatment (surgery, RT, chemotherapy, or hormone therapy). There was no analysis regarding delays with initial findings (either palpable masses or abnormal screening mammogram), diagnostic mammogram, RT treatment time, or total care-path time.

Ours is the first study to show an association between language and delays in pre-RT breast cancer care. In addition, it is the first study to examine both the interval from initial findings to diagnosis and the interval from diagnosis to key treatment points (including surgery and RT), making it the most comprehensive study examining language and delays in care. Our study revealed both statistically and clinically significant delays for the NES population in total care-path time and, more specifically, at 2 pre-RT timepoints: from initial findings to diagnostic mammogram, and from biopsy to surgical resection. Two of these intervals were specific to NES Latina patients, and the other (biopsy to surgical resection) applied to all NES patients. Strikingly, NES Latina patients had anywhere from a 4.7to 6.0-month longer total care-path time than any other population, including ES Latina patients. This difference is a significant clinical interval because a multi-month delay indicates the likelihood of significant barriers, whether personal, systemic, or a combination. Notably, there were no significant language delays immediately leading up to or within radiation treatment, indicating that radiation oncologists were providing timely care not associated with language interference. However, there were some race/ethnicity-specific delays at these timepoints that remain to be further investigated. For NES patients, delays were localized at the pre-RT timepoints. The total care-path delay was mainly attributed to the interval from initial findings to diagnostic mammogram, in which NES Latina patients waited an average of 6

\section{Table 2. Time in Months for NES Latina Patients Versus ES Latina and White Patients}

\begin{tabular}{|c|c|c|c|c|c|}
\hline Interval $^{a}$ & $\begin{array}{l}\text { NES Latina } \\
\text { Patients }\end{array}$ & $\begin{array}{l}\text { ES Latina } \\
\text { Patients }\end{array}$ & $\begin{array}{l}\text { Difference } \\
(P \text { Value })^{a}\end{array}$ & $\begin{array}{l}\text { ES White } \\
\text { Patients }\end{array}$ & $\begin{array}{l}\text { Difference } \\
\left(^{(P \text { Value })^{a}}\right.\end{array}$ \\
\hline Total care-path time & 13.53 & 7.56 & $5.97(.001)$ & 7.73 & $5.80(<.0001)$ \\
\hline Initial findings to diagnostic mammogram & 6.09 & 0.93 & $5.16(.0006)$ & 1.50 & $4.59(<.0001)$ \\
\hline
\end{tabular}

Bold indicates statistically significant $P$ value.

NES Latina patients experienced delays compared with ES Latina and ES White patients. The table highlights the 2 intervals that were statistically

significant: total care-path time and initial findings to diagnostic mammogram.

Abbreviations: ES, English-speaking; NES, non-English speaking.

${ }^{a}$ Statistical methods: time intervals are predicted means from the mixed-effects model, with accompanying $P$ values. 


\begin{tabular}{|lllll|}
\hline \multicolumn{2}{|l}{ Table 3. Time in Months for NES Versus ES Patients Along Breast Cancer Care Path } \\
\hline Interval & NES Patients & ES Patients & Difference & P Value \\
\hline Total care-path time $^{\text {a }}$ & 9.71 & 8.18 & 1.53 & .083 \\
\hline Initial findings to diagnostic mammogram $^{\mathrm{a}}$ & 3.00 & 1.60 & 1.40 & .055 \\
\hline Diagnostic mammogram to biopsy & 0.47 & 0.49 & -0.02 & .880 \\
\hline Biopsy to resection & 2.61 & 2.20 & 0.41 & .044 \\
\hline Resection to start of RT & 2.63 & 3.08 & -0.45 & .108 \\
\hline Start of RT to end of RT & 0.92 & 0.89 & 0.03 & .621 \\
\hline
\end{tabular}

Bold indicates statistically significant $P$ value.

NES patients experienced significant delays compared with ES patients between initial findings and diagnostic mammogram, and between biopsy and resection. These numbers are not specific to race/ethnicity.

Abbreviations: ES, English-speaking; NES, non-English-speaking; RT, radiotherapy

${ }^{a}$ Statistical methods: time intervals are predicted means from the mixed-effects model, with accompanying $P$ values.

months versus 0.6 to 3.0 months for other patients. Notably, a difference was seen at this interval between palpable versus screening presentation: more NES patients presented with palpable findings. This difference should be the focus of future interventions-targeting the NES patient population for screening mammograms may translate to decreased time before diagnostic mammogram and biopsy, and assistance for NES patients to enter into the medical system sooner for treatment.

Due to multifactorial reasons, including cultural beliefs, fear, socioeconomic status, and lack of health insurance, Latina women have experienced difficulty navigating the healthcare system, resulting in delays in care. ${ }^{34-36}$ Our study showed one interval in which all Latina patients experienced a delay (resection to start of RT), but otherwise the findings were specific for NES Latina patients. ES Latina patients fared generally well compared with other subgroups. NES Latina patients are often less acculturated and have shown a decreased knowledge of breast cancer and decreased compliance with screening. ${ }^{37,38}$ Strategies to address cultural beliefs, knowledge of breast cancer, and screening may help bring NES Latina patients into the system for more timely care.

Out of the 4.7- to 6-month total care-path delay, approximately 0.4 months were attributed to a delay for NES patients once they were within the medical system but pre-RT, between biopsy and resection. Some studies have shown a lack of clarity in communication for NES Latina patients during their breast cancer consultations; this issue is a potential target for addressing delays after diagnosis. ${ }^{39}$ In addition, successful patient navigation, including coordinating appointments, transportation, telephone support, interpretation, and assistance with insurance paperwork, is another target that has been shown to decrease delays in breast cancer treatment. ${ }^{40}$

The influence of language on delays in breast cancer care is particularly important in the United States, where the use of languages other than English increased by $148 \%$ from 1980 to 2009, especially Spanish, Vietnamese, and Russian. ${ }^{18}$ Highlighting the issues surrounding language as a health disparity is important given the paucity of other research investigating this concern. This is a hypothesisgenerating study, and the findings pose a potential platform for interventions targeting NES Latina patients pre-RT to allow for quicker access to diagnostic mammograms and to assist with their transition from biopsy to resection. Other factors that may need to be addressed for an effective intervention include health literacy, access to care, fear and cultural beliefs, or internal medical system issues involving interpreters and communication. Future studies could be aimed at qualitatively assessing barriers to care, such as via surveys or outreach to examine culture, religion, education, cost of care, and transportation in regard to NES patient treatment times.

We acknowledge the limitations of our study, which include its retrospective nature, its sample size with 2 years of data, and the select patient population. Given the RT population, patients largely underwent lumpectomy $(96.9 \%)$ rather than mastectomy. This decision regarding mastectomy versus breast-conserving surgery can take considerable time. Our data do not include the number of consultations with a surgeon, or whether a patient was delayed because she was considering mastectomy. Future studies could elucidate more details for this interval between biopsy and resection.

We also acknowledge the limitations of the propensity score matching, which may leave room for bias if crucial variables are left out. The most important factors (language, race/ethnicity, and insurance) were already included as individual predictors in the mixed-effects model. However, there remained variables, such as income/socioeconomic status, that were not available in our data and could contribute to delays. Note that insurance often serves as a proxy for these factors and was included in the mixed-effects model without any significant associated findings, but it could be limited by the 
sample size. Other limitations of propensity score matching often occur when stratification or the nearest neighbor are used for matching, but this study used exact matching to circumvent those issues.

Furthermore, we acknowledge that English language proficiency exists on a spectrum, but it was not possible to discern varying levels of proficiency in this retrospective analysis. This element is something that could be evaluated in future prospective language studies. Finally, we acknowledge the geographic limitations of this study, given that the cohort was from an academic institution in California. The NES patient representation in both the state of California and this study population was higher than the national average, and there were differences in the distribution of languages compared with national distribution. With regard to insurance and population health, California is one of the 39 states that accepted Medicaid expansion under the Affordable Care Act, and California is ranked in the top 10 US states for healthcare. ${ }^{41}$ For these reasons, findings are not directly generalizable to the nation but must instead be viewed as hypothesisgenerating for future work on a larger scale that addresses these national differences.

\section{Conclusions}

Language is associated with pre-RT delays in breast cancer care, specifically for NES Latina patients. Notably, there is a statistically and clinically significant delay in care for NES Latina patients of 5 to 6 months throughout their breast cancer care path. This delay is mainly attributed to the time before diagnostic mammogram; however, a delay also exists before resection. ES Latina patients did not experience similar delays, supporting the idea that race/ethnicity is not a proxy for language and that the 2 need to be separately included in disparity studies. Future work should target NES Latina patients to assist with their progress along the pre-RT breast cancer care path.

Submitted September 1, 2020; final revision received November 20, 2020; accepted for publication December 14, 2020.

Published online August 10, 2021.

Author contributions: Study concept: Balazy, Benitez, Horst. Data curation: Balazy, Benitez, Gutkin, Jacobson, von Eyben. Investigation: Balazy, Benitez, von Eyben, Horst. Methodology: Balazy, Benitez, von Eyben, Horst. Formal analysis: von Eyben. Project administration: Balazy, Benitez, Gutkin, Jacobson, Horst. Resources: All authors. Software: von Eyben. Validation: Balazy, von Eyben, Horst. Visualization: Balazy, Benitez, von Eyben, Horst. Supervision: Horst. Writing-original draft: Balazy. Writing—review and editing: Balazy, Benitez, Gutkin, von Eyben, Horst.

Disclosures: The authors have disclosed that they have not received any financial consideration from any person or organization to support the preparation, analysis, results, or discussion of this article.

Funding: Cecil M. Benitez, MD, PhD, was supported by Medical Scholars funding through the Stanford School of Medicine.

Correspondence: Kathleen C. Horst, MD, Department of Radiation Oncology, Stanford Comprehensive Cancer Center, Stanford University School of Medicine, 875 Blake Wilbur Drive, Stanford, CA 94305-5847. Email: kateh@stanford.edu

\section{References}

1. American Cancer Society. Cancer Facts \& Figures 2020. Accessed December 23, 2020. Available at: https://www.cancer.org/content/dam/ cancer-org/research/cancer-facts-and-statistics/annual-cancer-facts-andfigures/2020/cancer-facts-and-figures-2020.pdf

2. Buja A, Lago L, Lago S, et al. Marital status and stage of cancer at diagnosis: a systematic review. Eur J Cancer Care (Engl) 2018;27:e12755.

3. Du Xianglin L, Gor BJ. Racial disparities and trends in radiation therapy after breast-conserving surgery for early-stage breast cancer in women, 1992 to 2002. Ethn Dis 2007;17:122-128.

4. Ellis L, Canchola AJ, Spiegel D, et al. Trends in cancer survival by health insurance status in California from 1997 to 2014. JAMA Oncol 2018;4:317-323.

5. Mobley LR, Kuo TMM, Watson L, et al. Geographic disparities in late-stage cancer diagnosis: multilevel factors and spatial interactions. Health Place 2012;18:978-990.

6. Thomson GE, Mitchell F, Williams M. Examining the Health Disparities Research Plan of the National Institutes of Health: Unfinished Business. Washington, DC: National Academies Press; 2006.

7. Silber JH, Rosenbaum PR, Ross RN, et al. Disparities in breast cancer survival by socioeconomic status despite Medicare and Medicaid insurance. Milbank O 2018;96:706-754.

8. Yedjou CG, Tchounwou PB, Payton M, et al. Assessing the racial and ethnic disparities in breast cancer mortality in the United States. Int J Environ Res Public Health 2017;14:486.

9. Newman LA. Breast cancer disparities: socioeconomic factors versus biology. Ann Surg Oncol 2017;24:2869-2875.

10. Warner ET, Tamimi RM, Hughes ME, et al. Racial and ethnic differences in breast cancer survival: mediating effect of tumor characteristics and sociodemographic and treatment factors. J Clin Oncol 2015;33:2254-2261.

11. Vona-Davis L, Rose DP. The influence of socioeconomic disparities on breast cancer tumor biology and prognosis: a review. J Womens Health (Larchmt) 2009;18:883-893.
12. Nonzee NJ, Ragas DM, Ha Luu T, et al. Delays in cancer care among lowincome minorities despite access. J Womens Health (Larchmt) 2015;24: 506-514.

13. Khanna S, Kim KN, Qureshi MM, et al. Impact of patient demographics, tumor characteristics, and treatment type on treatment delay throughout breast cancer care at a diverse academic medical center. Int J Womens Health 2017;9:887-896.

14. McGee SA, Durham DD, Tse CK, et al. Determinants of breast cancer treatment delay differ for African American and White women. Cancer Epidemiol Biomarkers Prev 2013;22:1227-1238.

15. Hershman DL, Wang $X, M c B r i d e ~ R$, et al. Delay in initiating adjuvant radiotherapy following breast conservation surgery and its impact on survival. Int J Radiat Oncol Biol Phys 2006;65:1353-1360.

16. Punglia RS, Saito AM, Neville BA, et al. Impact of interval from breast conserving surgery to radiotherapy on local recurrence in older women with breast cancer: retrospective cohort analysis. BMJ 2010; 340:c845.

17. Balazy KE, Benitez CM, Gutkin PM, et al. Association between primary language, a lack of mammographic screening, and later stage breast cancer presentation. Cancer 2019;125:2057-2065.

18. Ortman JM, Shin HB. Language projections: 2010 to 2020. United States Census Bureau. Accessed December 24, 2020. Available at: https://www.census.gov/library/working-papers/2011/demo/2011Ortman-Shin.html

19. Rose DE, Tisnado DM, Malin JL, et al. Use of interpreters by physicians treating limited English proficient women with breast cancer: results from the provider survey of the Los Angeles Women's Health Study. Health Serv Res 2010;45:172-194.

20. Karliner LS, Hwang ES, Nickleach D, et al. Language barriers and patientcentered breast cancer care. Patient Educ Couns 2011;84:223-228.

21. Migration Policy Institute. California: language \& education. Accessed December 24, 2020. Available at: https://www.migrationpolicy.org/data/ state-profiles/state/language/CA 
22. Center for Immigration Studies. 67.3 million in the United States spoke a foreign language at home in 2018. Accessed October 30, 2020. Available at: https://cis.org/Report/673-Million-United-States-Spoke-ForeignLanguage-Home-2018

23. United States Census Bureau. Language spoken at home. Accessed October 30, 2020. Available at: https://data.census.gov/cedsci/ table $? \mathrm{q}=$ language\&tid=ACSST1Y2019.S1601\&hidePreview $=$ true

24. Kaiser Family Foundation. Status of state Medicaid expansion decisions: interactive map. Accessed November 3, 2020. Available at: https://www. $\mathrm{kff.org} / \mathrm{medicaid} /$ issue-brief/status-of-state-medicaid-expansiondecisions-interactive-map/

25. Kaiser Family Foundation. The California Health Care Landscape. Accessed on November 3, 2020. Available at: https://www.kff.org/health-reform/ fact-sheet/the-california-health-care-landscape/

26. Dong J, Esham KS, Boehm L, et al. Timeliness of treatment initiation in newly diagnosed patients with breast cancer. Clin Breast Cancer 2020;20: e27-35.

27. Smith EC, Ziogas A, Anton-Culver H. Delay in surgical treatment and survival after breast cancer diagnosis in young women by race/ethnicity. JAMA Surg 2013;148:516-523.

28. Ho PJ, Cook AR, Binte Mohamed Ri NK, et al. Impact of delayed treatment in women diagnosed with breast cancer: a population-based study. Cancer Med 2020;9:2435-2444.

29. Polverini AC, Nelson RA, Marcinkowski E, et al. Time to treatment: measuring quality breast cancer care. Ann Surg Oncol 2016;23:3392-3402.

30. Bleicher RJ, Ruth K, Sigurdson ER, et al. Time to surgery and breast cancer survival in the United States. JAMA Oncol 2016;2:330-339.

31. Li Y, Zhou Y, Mao F, et al. The influence on survival of delay in the treatment initiation of screening detected non-symptomatic breast cancer. Sci Rep 2019;9:10158.
32. Warner ET, Tamimi RM, Hughes ME, et al. Time to diagnosis and breast cancer stage by race/ethnicity. Breast Cancer Res Treat 2012;136: 813-821.

33. Gorin SS, Heck JE, Cheng B, et al. Delays in breast cancer diagnosis andtreatment by racial/ethnic group. Arch Intern Med 2006;166: 2244-2252.

34. Tejeda S, Gallardo RI, Ferrans CE, et al. Breast cancer delay in Latinas: the role of cultural beliefs and acculturation. J Behav Med 2017;40: 343-351.

35. Tejeda S, Thompson B, Coronado GD, et al. Barriers and facilitators related to mammography use among lower educated Mexican women in the USA. Soc Sci Med 2009;68:832-839.

36. Martínez ME, Gomez SL, Tao L, et al. Contribution of clinical and socioeconomic factors to differences in breast cancer subtype and mortality between Hispanic and non-Hispanic white women. Breast Cancer Res Treat 2017;166:185-193.

37. Garcia RZ, Carvajal SC, Wilkinson AV, et al. Factors that influence mammography use and breast cancer detection among Mexican-American and African-American women. Cancer Causes Control 2012;23:165-173.

38. Abraído-Lanza AF, Chao MT, Gates CY. Acculturation and cancer screening among Latinas: results from the National Health Interview Survey. Ann Behav Med 2005;29:22-28.

39. Schapira MM, Faghri A, Jacobs EA, et al. Communication and shared decision making in the breast cancer treatment consultation: a comparative analysis of English- and Spanish-speaking patients. MDM Policy Pract 2019 4:2381468319881651.

40. Ramirez A, Perez-Stable E, Penedo F, et al. Reducing time-to-treatment in underserved Latinas with breast cancer: the Six Cities Study. Cancer 2014 120:752-760.

41. U.S. News \& World Report. Health care rankings. Accessed November 3, 2020. Available at https://www.usnews.com/news/best-states/rankings/ health-care 


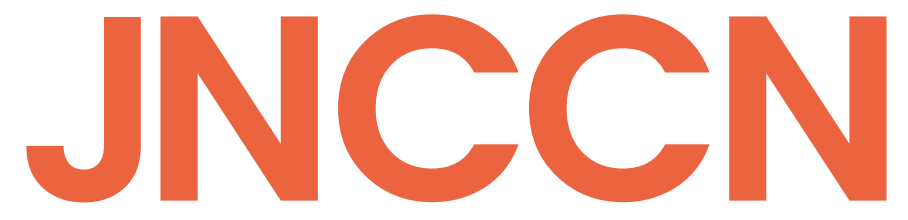

(n)

Supplemental online content for:

\section{Delays in Care Associated With Non-English-Speaking Patients With Breast Cancer}

Katy E. Balazy, MD, MPH; Cecil M. Benitez, MD, PhD; Paulina M. Gutkin, BS; Clare E. Jacobson, BA; Rie von Eyben, MS; and Kathleen C. Horst, MD

J Natl Compr Canc Netw, doi: 10.6004/jnccn.2020.7797

eFigure 1: Matched Cohort Diagram for NES to ES Patients

eTable 1: Distribution of Languages in Matched NES Patients

eTable 2: Statistical Model Analyzing Delays in Care at Each Time Interval

eTable 3: Time in Months for NES Versus ES at Each Time Interval

eTable 4: Time in Months for Each Race/Ethnicity at Each Time Interval

eTable 5: Time in Months for NES Versus ES Patients by Race/Ethnicity at Each Time Interval

eTable 6: Time in Months for Public Versus Private Insurance at Each Time Interval 


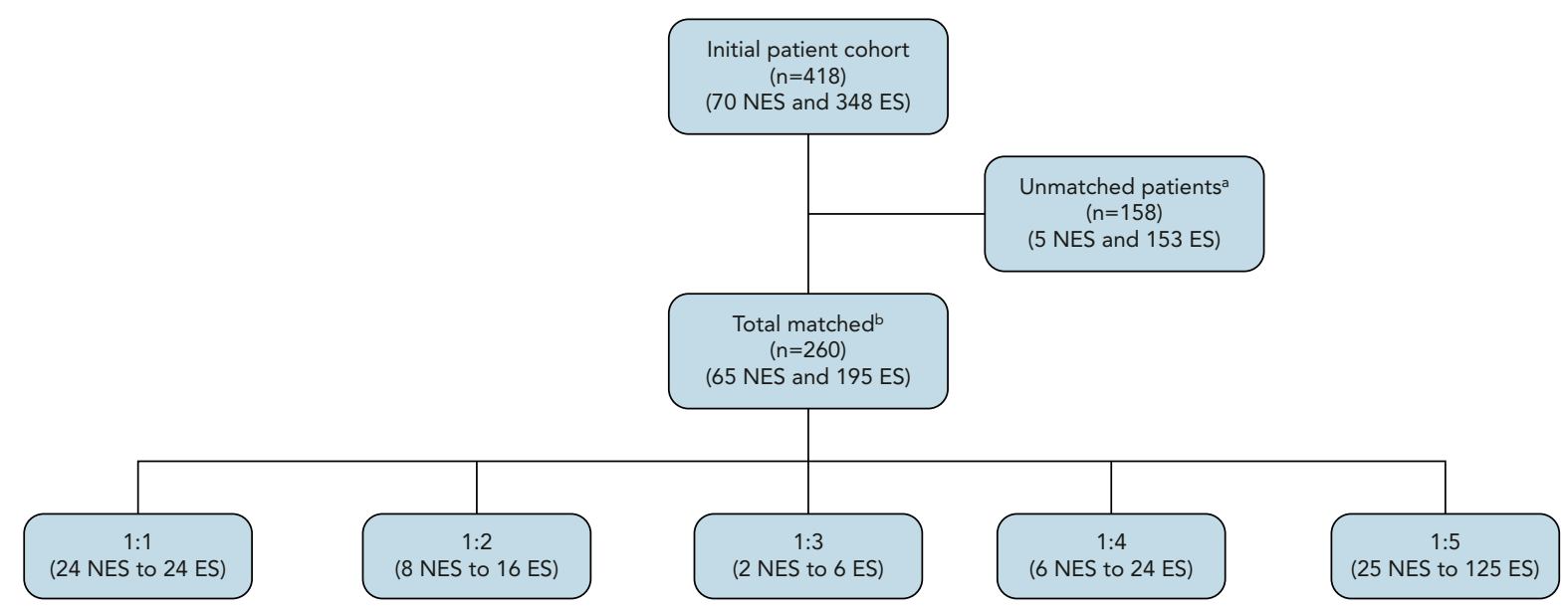

eFigure 1. Matched cohort diagram for NES to ES patients.

Abbreviations: ES, English-speaking; NES, non-English-speaking.

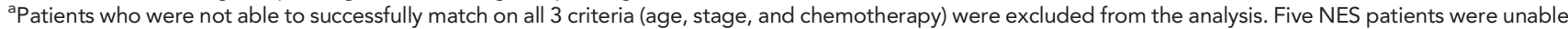
to match to ES patients within 5 years of age and were excluded. There were only 4 patients with stage IV disease, and all were ES patients; none were included because there were no NES patients with stage IV disease.

${ }^{b}$ Matching was performed using propensity score matching where each NES patient was matched to at most 5 ES patients. The model required an exact match on both stage and chemotherapy status. After matching, the difference in age was computed and all pairs with an age difference $>5$ years were excluded. 


\section{eTable 1. Distribution of Languages in Matched}

\section{NES Patients}

\begin{tabular}{|ll|}
\hline Language & $\mathbf{n}$ \\
\hline Burmese & 1 \\
\hline Cantonese & 2 \\
\hline English & 0 \\
\hline Farsi & 4 \\
\hline French & 1 \\
\hline Hindi & 1 \\
\hline Hungarian & 1 \\
\hline Korean & 3 \\
\hline Mandarin & 9 \\
\hline Polish & 1 \\
\hline Portuguese & 2 \\
\hline Punjabi (Panjabi) & 1 \\
\hline Russian & 1 \\
\hline Spanish & 28 \\
\hline Tagalog & 1 \\
\hline Vietnamese & 8 \\
\hline Other & 1 \\
\hline Total & 65 \\
\hline
\end{tabular}

Abbreviation: NES, non-English-speaking.

\section{eTable 2. Statistical Model ${ }^{\mathrm{a}}$ Analyzing Delays in Care at Each Time Interval}

\begin{tabular}{|lllllll|}
\hline & $\begin{array}{l}\text { Initial Findings to } \\
\text { Diagnostic } \\
\text { Mammogram } \\
(P \text { Value })\end{array}$ & $\begin{array}{l}\text { Diagnostic } \\
\text { Mammogram to } \\
\text { Biopsy } \\
(P \text { Value })\end{array}$ & $\begin{array}{l}\text { Biopsy to } \\
\text { Resection } \\
(P \text { Value })\end{array}$ & $\begin{array}{l}\text { Resection to } \\
\text { Start of RT } \\
(P \text { Value })\end{array}$ & $\begin{array}{l}\text { Start of RT to } \\
\text { End of RT } \\
(P \text { Value })\end{array}$ & $\begin{array}{l}\text { Total } \\
\text { Care-Path } \\
\text { Time } \\
(P \text { Value })\end{array}$ \\
\hline Race/Ethnicity & .150 & .329 & .690 & .035 & .022 & .121 \\
\hline Language & $.055^{\mathrm{b}}$ & .880 & .044 & .108 & .621 & .083 \\
\hline $\begin{array}{l}\text { Interaction between language } \\
\text { and race/ethnicity }\end{array}$ & .014 & .968 & .803 & .289 & .137 \\
\hline Insurance & .802 & .726 & .462 & .192 & .660 \\
\hline
\end{tabular}

Bold indicates statistically significant variables at each interval.

Abbreviations: ES, English-speaking. NES, Non-English-speaking; RT, radiotherapy.

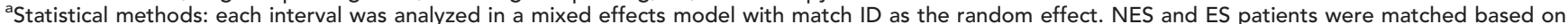
cancer stage, chemotherapy, and age ( \pm 5 years). Then individual predictors of language, insurance, and race/ethnicity were included in the model.

${ }^{b}$ All 2-way interactions between race/ethnicity, language, and insurance were examined. Only the interaction of language and race/ethnicity was included in the model because all others were not significant. 


\section{eTable 3. Time in Months for NES Versus ES at Each Time Interval}

\begin{tabular}{|lll|}
\hline Interval & NES & ES Value \\
\hline Total care-path time & 9.71 & 8.18 \\
\hline Initial findings to diagnostic mammogram & 3.00 & 1.60 \\
\hline Diagnostic mammogram to biopsy & 0.47 & 0.49 \\
\hline Biopsy to resection & 2.61 & 2.20 \\
\hline Resection to start of RT & 2.63 & 3.08 \\
\hline Start of RT to end of RT & .055 & .044 \\
\hline
\end{tabular}

Bold indicates statistically significant $P$ value.

Abbreviations: ES, English-speaking. NES, Non-English-speaking; RT, radiotherapy.

\begin{tabular}{|c|c|c|c|c|c|}
\hline Interval & White & Asian & Latina & Black/Other & $P$ Value \\
\hline Total care-path time & 7.60 & 8.40 & 10.55 & 9.22 & .121 \\
\hline Initial findings to diagnostic mammogram & 1.05 & 2.30 & 3.51 & 2.30 & .150 \\
\hline Diagnostic mammogram to biopsy & 0.33 & 0.45 & 0.74 & 0.41 & .329 \\
\hline Biopsy to resection & 2.48 & 2.39 & 2.20 & 2.56 & .690 \\
\hline Resection to start of RT & 2.76 & 2.60 & 3.58 & 2.76 & .035 \\
\hline Start of RT to end of RT & 0.91 & 0.97 & 0.77 & 1.00 & .022 \\
\hline
\end{tabular}

Bold indicates statistically significant $P$ value.

Abbreviation: RT, radiotherapy

\begin{tabular}{|c|c|c|c|c|c|c|c|c|c|}
\hline \multirow[b]{2}{*}{ Interval } & \multicolumn{4}{|c|}{ NES } & \multicolumn{4}{|c|}{ ES } & \multirow[b]{2}{*}{$P$ Value } \\
\hline & White & Asian & Latina & Black/Other & White & Asian & Latina & Black/Other & \\
\hline Total care-path time & 7.48 & 8.25 & 13.53 & 9.59 & 7.73 & 8.56 & 7.56 & 8.86 & .024 \\
\hline Initial findings to diagnostic mammogram & 0.61 & 2.27 & 6.09 & 3.02 & 1.50 & 2.34 & 0.93 & 1.61 & .014 \\
\hline Diagnostic mammogram to biopsy & 0.25 & 0.44 & 0.78 & 0.42 & 0.42 & 0.45 & 0.71 & 0.40 & .968 \\
\hline Biopsy to resection & 2.84 & 2.61 & 2.29 & 2.70 & 2.11 & 2.16 & 2.17 & 2.40 & .803 \\
\hline Resection to start of RT & 2.71 & 2.55 & 3.52 & 1.73 & 2.92 & 2.71 & 3.54 & 3.13 & .289 \\
\hline Start of RT to end of RT & 0.97 & 0.88 & 0.84 & 0.99 & 0.91 & 1.02 & 0.61 & 1.01 & .137 \\
\hline
\end{tabular}

Bold indicates statistically significant $P$ value.

Abbreviations: ES, English-speaking. NES, Non-English-speaking; RT, radiotherapy. 
4 - Balazy et al

\section{eTable 6. Time in Months for Public Versus Private Insurance at Each Time Interval}

Interval

Total care-path time

Initial findings to diagnostic mammogram

Diagnostic mammogram to biopsy

Biopsy to resection

Resection to start of RT

Start of RT to end of RT

Abbreviation: RT, radiotherapy
Public Insurance

\begin{tabular}{lll}
8.97 & 8.91 & .937 \\
2.24 & 2.37 & .802 \\
0.46 & 0.51 & .726 \\
2.48 & 2.33 & .462 \\
3.02 & 2.68 & .192 \\
0.89 & 0.92 & .660 \\
\hline
\end{tabular}

